\title{
High Resolution Ultrasonic Imaging in the Evaluation of the Posterior Segment Disorders
}

\author{
Magdy E. Khallaf, Fatma A Atwa, Abd Allah El Husiny, Ahmad M Salah Eldein \\ Department of Ophthalmology, Faculty of Medicine, Al-Azhar University, Cairo, Egypt \\ Corresponding author: Ahmad Mohammad Salah Eldein, E-mail: dr_ahmad_aa@yahoo.com, Phone no.: 01005422240
}

\begin{abstract}
Purpose: to evaluate the role of high resolution $20 \mathrm{MHz}$ ultra sound in assessment of posterior segment disorders.

Patients and Methods: a prospective, case series study was conducted on 40 patients who attended the ophthalmology outpatient clinic of Al-Azhar university hospitals (2014-2018). All should have a posterior segment disorder either retinal, vascular, vitreo-macular, optic nerve head or choroidal disorder. They should be with clear media, therefor we can reach a diagnosis based on clinical examination and augmented by optical coherence tomography (OCT) and fundus fluorescein angiography (FFA) whenever indicated, the patients were evaluated using both high resolution $20 \mathrm{MHz}$ ultrasound(US) and conventional $12.5 \mathrm{MHz}$ US and compare their results to the gold standard clinical diagnosis to assess their sensitivity to reach the diagnosis.
\end{abstract}

Results \& Conclusion: we have two main characters for well diagnosis by any US tool, resolution and sensitivity, and neither the $20 \mathrm{MHz}$ nor $12.5 \mathrm{MHz}$ could combine both of them. Therefore, we recommended tocombine the examination by both of them as they are complementary to each other.

Keywords: High Resolution US, OCT, FFA, 20MHz US

\section{INTRODUCTION}

Ultrasonography is one of the most valuable diagnostic tool in ophthalmology. It is a safe, reliable, non-invasive diagnostic tool that accepted as an integral tool in diagnosis and management of a wide spectrum of intra ocular and orbital pathologies even in opaque media as corneal and anterior chamber opacities, cataract and vitreous hemorrhage ${ }^{(\mathbf{1})}$.

Developments in transducer, pulser/receiver, and digital signal-processing technologies particularly development of sensitive, broadband $20-\mathrm{MHz}$ transducers have allowed higher frequencies to be valuable for evaluation of the posterior segment ${ }^{(2)}$.

OCT had filled the gap in high-resolution imaging of the posterior segment of the eye, which is more valuable than resolution provided by $10-\mathrm{MHz}$ US, it can provides an axial resolution of $10 \mu \mathrm{m}$ and a lateral resolution of $20 \mu \mathrm{m}$, however OCT can be used only when all optical media (cornea, lens, vitreous) are mostly clear and is limited to the central fundus (i.e., images are acquired through the pupil) ${ }^{(3)}$.

Recently, the availability of higher frequency probes such as the $20-\mathrm{MHz}$ has made the evaluation of the posterior segment structures much better with higher resolution in comparison to the previous $10-\mathrm{MHz}$ probe ${ }^{(4)}$.

\section{AIM OF THE WORK}

To evaluate the use of higher frequency ultrasound to provide improved resolution of the posterior segment in comparison to the conventional frequencies in clear media, keeping the clinical diagnosis augmented by OCT \& FFA as the gold standard, therefore we can assess the high frequency US reliability and dependability in opaque media.

\section{PATIENTS AND METHODS}

The study protocol adhered to the tenets of Helsinki and was approved by ethical board of Al-Azhar uniVersity and informed written consent was taken from each patient in the study, A prospective case series study was conducted on forty eyes of thirty-three patients who attended the ophthalmology outpatient clinic of Al-Azhar university hospitals (2014-2018) with different posterior segment disorders.

Inclusion criteria, All patients should have: 1- Cornea, anterior segment, crystalline lens and vitreous gel all are clear enough for fundus examination and OCT, FFA imaging. 2- Posterior segment pathology, that could be either posterior vitreal, retinal, optic nerve head or choroidal pathology. Uncooperative patients and patients who had unclear media (like central corneal opacities, dense cataract, dense vitreous hemorrhage, etc.) that prevent fundus viewing and (or) FFA or OCT. to be taken when been indicated for diagnosis were excluded from this study.

Each patient subjected to the following: 1Complete ophthalmological examination including 
best corrected visual acuity (BCVA) will be measured using a Snellen chart. Anterior segment examination using slit lamp biomicroscopy using (Haag-Streit, BM90, slit lamp, Haag-Streit ${ }^{\mathrm{TM}}$, Germany.). Intra ocular pressure (IOP) measurement by applanation tonometry. (Haag-Streit, AT900, applanation tonometry, Haag-Streit ${ }^{\mathrm{TM}}{ }^{\text {, Germany.). }}$ Dilated funduscopy will be performed using indirect ophthalmoscopy (Keeler ${ }^{\circledR M}$ Ltd, Windsor, UK) and stereoscopic biomicroscopy with a non-contact +90 or +78 diopter lens. 2- Ophthalmic ultrasound evaluation using two different techniques

A- First scan with an immersion technique using $20-\mathrm{MHz}$ High frequency ultrasound probe of OTI scan ${ }^{\circledR}$ (OTI, B\A high frequency ophthalmic ultrasound scan (OTI ophthalmic technologies inc toronto, canada). The patient lying supine and after instilling anesthetic drops, the appropriate shell size was chosen and placed beneath the eyelids and filled with methyl cellulose depending on the size of the shell, it may be necessary to allow fluid (normal saline) to overflow the edges of the shell to prevent air bubbles formation on the surface in front of the probe. For posterior segment evaluation, axial scans may not be optimal due to sound attenuation from the lens, Para axial scans facilitate the evaluation of the peripapillary area, longitudinal and transverse (cross section) through the macula was obtained by both high and medium gain settings then decrease the gain to evaluate the macular area and improving contrast.

B- Second B scan through closed eye lid using Conventional ultrasound $12.5-\mathrm{MHz}$ of (Mentor (®) Advent TM AlB system, mentor ophthalmic inc) The patient lying supine and the probe was placed over the closed eyelid at the temporal equator, aiming nasally and posteriorly after application of a coupling gel (Hydroxy- propyl methyl cellulose) to facilitate sound transmission and allow better contact of the surface of the probe with the eye lid. Fine adjustment of the probe was made while the patient fixated gaze at the primary position or slightly infero- temporally. Transverse (crosssection) and longitudinal (long-section) scans were performed on optic nerve head giving vertical and horizontal sections of the disc cup. B-scan examination was performed with medium gain setting; initially, we displayed the nerve at a high gain level then decreased the gain setting.

3- Optical coherence tomography (OCT) and (or) fundus fluorescin angiography (FFA) whenever indicated using Topcon triton swept source OCT \& fundus fluorescein angiography scan (Topcon® DRI OCT triton series, topcon corporation, Japan).

The patients were classified into four main groups: (I) Retinal and vascular disorders (17 eyes of15 patients) (II) Vitreo-macular interface disorders (6) eyes, (6) patients (III) Optic nerve head disorders (9) eyes (6) patients (IV) Choroidal disorders (8) eyes (6) patients.

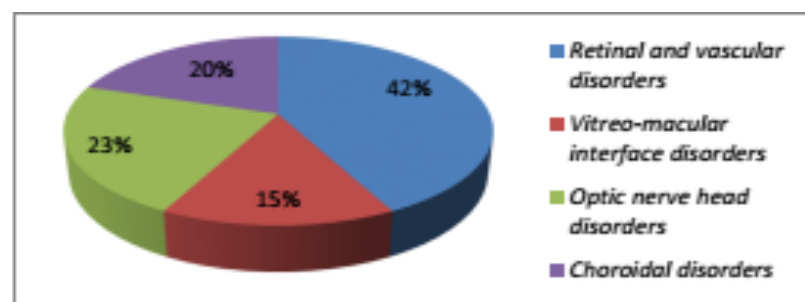

Fig. (1): Pie chart shows Classification of the study group according to clinical diagnosis.

\section{Statistical analysis:}

Analysis of data was performed using SPSS v. 25 (Statistical Package for Scientific Studies) for Windows \& MedCalc v. 18 and compared the output of each group by odds ratio and Forest plot, The significance of the results was assessed in the form of P-value that was differentiated into: non-significant when $\mathrm{P}$-value > 0.05 , significant when $\mathrm{P}$-value $\leq 0.05$, highly significant when $\mathrm{P}$-value $\leq 0.01$.

\section{RESULTS}

This study included forty eyes of thirty-three patients. 17 males and 16 females. The mean age was 36 years (range from 5 to 67 years); they were enrolled, as they were fitted in the inclusion criteria, and examined in our prospective case report study.

In group (I) According to pathology detection there was statistically no significant difference between both types of US and they were comparable to the clinical diagnosis which augmented by either OCT, FFA or both of them, However, according to the quality of the pathology detection there was statistically significant difference between clinical and both types of US with $(\mathrm{OR}=9.47, \mathrm{P} .<0.05)$ but still comparable to it. There was also statistically total insignificant increase of pathology qualification of Retinal and vascular disorders 1.533 times by $20 \mathrm{MHz}$ US more than by $12.5 \mathrm{MHzUS}(\mathrm{OR}=1.533 ; \mathrm{P}>0.05)$. Regarding retinal pathology quantification There was statistically total insignificant increase of pathology quantification of Retinal and vascular disorders 5 times by OCT-FFA more than by $20 \mathrm{MHz}$ US (OR=5, P.> 0.05). 
Table 1: Comparing pathology qualification of retinal and vascular disorders by each method of investigation using odds ratio and Forest plot in the study group ( $\mathrm{n}=17$ cases $)$

\begin{tabular}{|l|c|c|c|c|c|}
\hline \multirow{2}{*}{$\begin{array}{c}\text { Investigation } \\
\text { (Retinal\& vascular disorders) }\end{array}$} & \multicolumn{2}{|c|}{ Pathology qualification } & \multirow{2}{*}{\begin{tabular}{c}
\multirow{2}{*}{ 95\% CI } \\
(OR)
\end{tabular}} & \multirow{2}{*}{ P.value } \\
\cline { 2 - 3 } & Yes & No & $\mathbf{1 2 2 5}$ & 22.9 to 65270.9 & $\mathbf{0 . 0 0 0 5}$ \\
\hline OCT-FFA & $17 / 17$ & $0 / 17$ & 3.361 & 0.82 to 13.723 & 0.0912 \\
\hline 20 MHz US & $11 / 17$ & $6 / 17$ & 2.041 & 0.521 to 7.999 & 0.3060 \\
\hline Total (fixed effects) & $10 / 17$ & $7 / 17$ & $\mathbf{5 . 9 8 6}$ & 2.72 to 13.132 & $<\mathbf{0 . 0 0 1}$ \\
\hline Total (random effects) & $38 / 51$ & $13 / 51$ & 9.265 & 0.81 to 105.53 & 0.073 \\
\hline
\end{tabular}

- There was statistically significant increase of cases with pathology qualification of Retinal and vascular disorders by OCT-FFA more than cases without qualification $\mathrm{OR}=1225, \mathrm{P} .<0.05$ ).

- There was statistically insignificant increase of cases with pathology qualification of Retinal and vascular disorders by $12.5 \mathrm{MHz}$ and $20 \mathrm{MHz}$ US more than cases without qualification (P.>0.05).

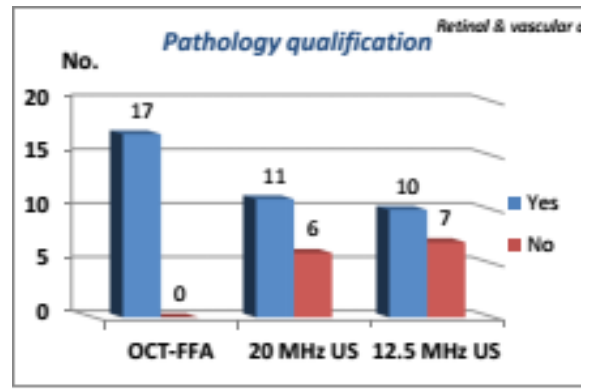

Fig. (2) Column chart comparing pathology qualification of retinal and vascular disorders by each method of investigation

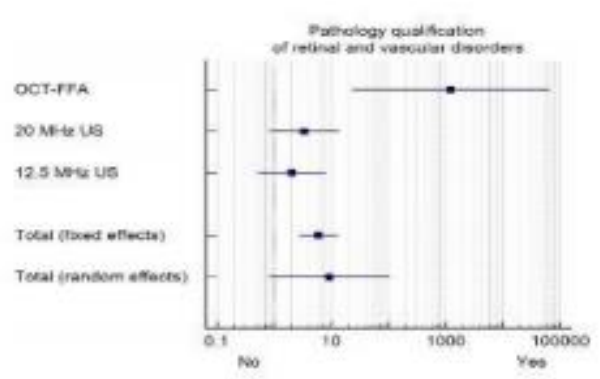

Fig. (3) Forest plot chart comparing pathology qualification of retinal and vascular disorders by each method of investigation.

\section{Case No. 1}

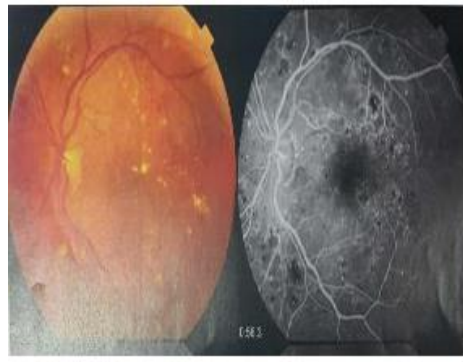

(A)

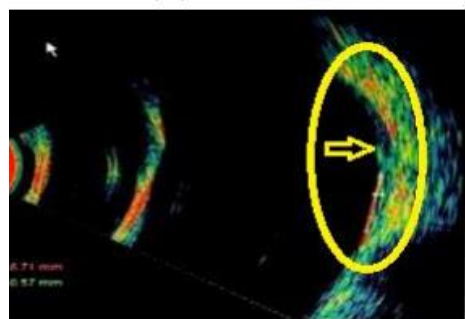

(C)

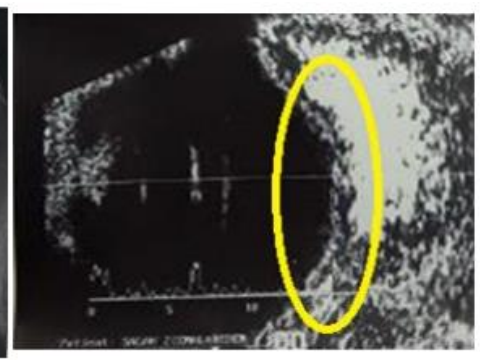

(B)

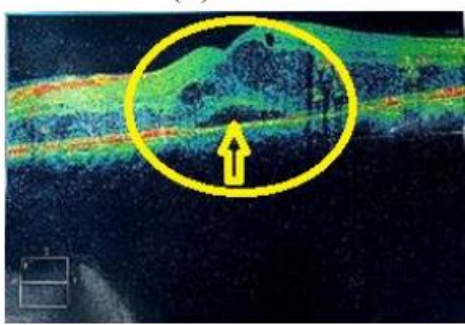

(D)

Figure 4: LT NPDR with CME and neurosensory detachment (A): FFA shows NPDR with DME (B): conventional $(12.5 \mathrm{MHz})$ UIS: Retinal fuzziness could be detected which indicates macular edema with demonstration of small cystic spaces within the lesion \{yellow circles\}. (C): longitudinal B-scan (20 MHz UIS) through the macula shows moderately elevated lesion that indicates DM \{yellow circles\}. In addition to an area of neurosensory detachment that could be seen clearly (yellow arrow) with central macular thickness $\pm 570 \mu \mathrm{m}$ (D) OCT shows CME with evidence of NSD with central macular thickness measuring $611 \mu \mathrm{m}$, with thickness difference $\pm 49 \mu \mathrm{m}$ than that measured by US. 


\section{Case No. 2}

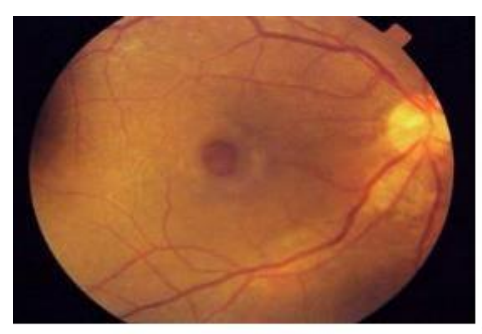

(A)

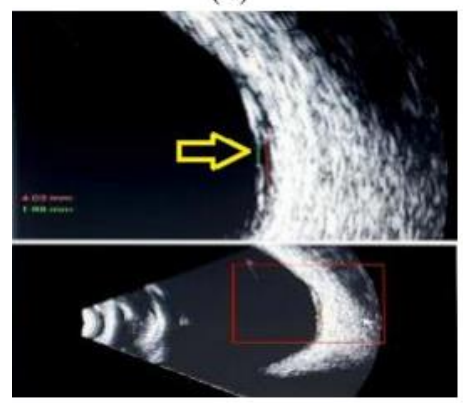

(C)

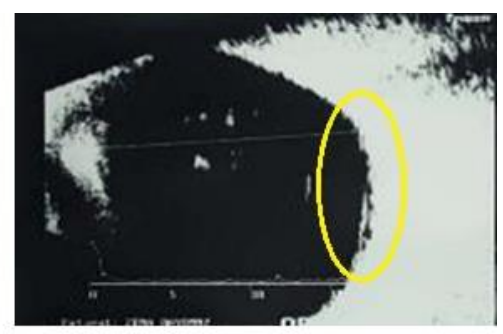

(B)

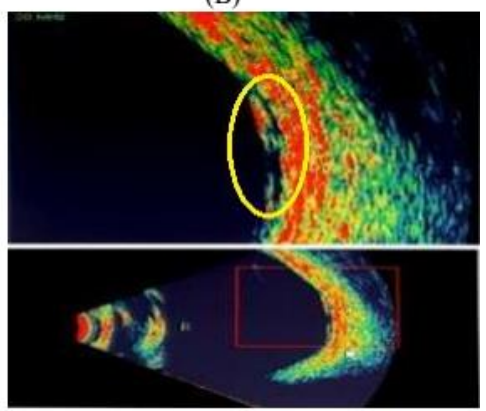

(D)

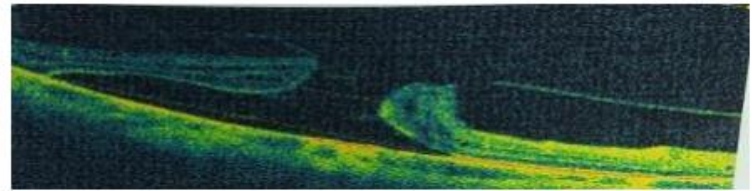

(E)

Figure 5: RT full thickness Macular hole (A) Colored fundus photo shows loss of foveal reflex with large macular hole (B) Para axial B-scan (12.5MHz) displaying a hole with overlying partial PVD (C) \& (D): reverse longitudinal (20 MHz) B-scan at reduced gain demonstrates full thickness macular hole with shallow RD, although it didn't show the Vitero macular traction well as being in the OCT fig (E), but still superior in resolution and tissue differentiation than conventional US fig (b). There some differences in operculum and hole base measurement, as by $20 \mathrm{MHz}$ UIS the operculum measurement was $1.95 \mathrm{~mm}$, and the base measured $4.09 \mathrm{~mm}$, while by OCT it's operculum measured $760 \mu \mathrm{m}$, and it's base was $3.43 \mathrm{~mm}$.

In group no. (II) patients with (Vitreo-macular interface disorders) we found that:

While assessment of the posterior vitreous, presence or absence of ERM, presence of any vitreous floaters or subhyaloid hemorrhage, the $12.5 \mathrm{MHz}$ US was more valuable than $20 \mathrm{MHz}$, although it was statistically insignificant $(\mathrm{OR}=2.231 ; \mathrm{P}>0.05)$.

On assessment of the retinal tissue itself, macular contour and vitreomacular interface, the resolution power and well tissue differentiation of $20 \mathrm{MHz}$ US was more beneficial, the difference between both was also statistically insignificant $(\mathrm{OR}=0.448 ; \mathrm{P}>0.05)$ however, practically the difference was obvious.

Table 2: Comparing Posterior vitreous lesion qualification of vitreo macular disorders by each method of investigation using odds ratio and Forest plot in the study group ( $\mathrm{n}=6$ cases)

\begin{tabular}{|l|c|c|c|c|c|}
\hline \multirow{2}{*}{\begin{tabular}{c}
\multirow{2}{*}{$\begin{array}{c}\text { Investigation } \\
\text { (Vitreo macular disorders) }\end{array}$} \\
\cline { 2 - 3 }
\end{tabular}} & \multicolumn{2}{|c|}{$\begin{array}{c}\text { Posterior vitreous } \\
\text { lesion qualification }\end{array}$} & \multirow{2}{*}{ Odds ratio (OR) } & \multirow{2}{*}{ 95\% CI } & \multirow{2}{*}{ P.value } \\
\cline { 2 - 3 } & Yes & No & & & \\
\hline OCT-FFA & $6 / 6$ & $0 / 6$ & 169 & 2.89 to 9876.1 & $\mathbf{0 . 0 1 3 4}$ \\
\hline 20 MHz US & $4 / 6$ & $2 / 6$ & 4 & 0.36 to 44.115 & 0.2577 \\
\hline $12.5 \mathrm{MHz}$ US & $6 / 6$ & $0 / 6$ & 169 & 2.89 to 9876.1 & $\mathbf{0 . 0 1 3 4}$ \\
\hline Total (fixed effects) & $16 / 18$ & $2 / 18$ & 19.968 & 3.94 to 101.11 & $<\mathbf{0 . 0 0 1}$ \\
\hline Total (random effects) & $16 / 18$ & $2 / 18$ & 31.231 & 1.99 to 488.59 & 0.014 \\
\hline
\end{tabular}


Magdy E. Khallaf et al.

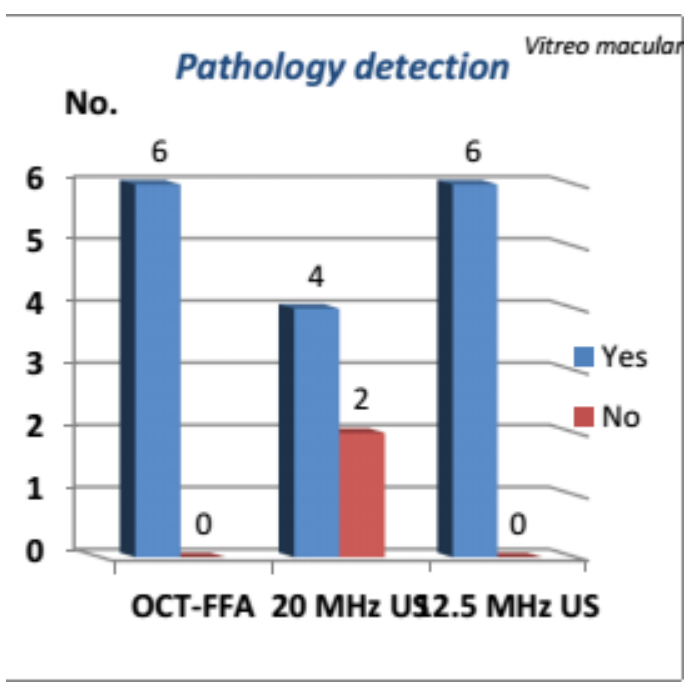

Fig. (6). Column chart comparing pathology detection of vitreo macular disorders by each method of investigation

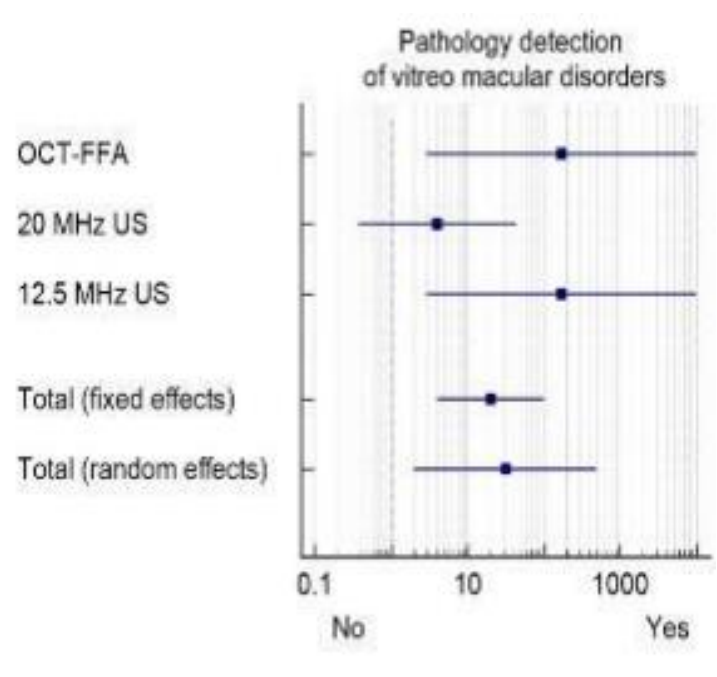

Fig. (7). Forest plot chart comparing pathology detection of vitreo macular disorders by each method of investigation

\section{Case No.5}
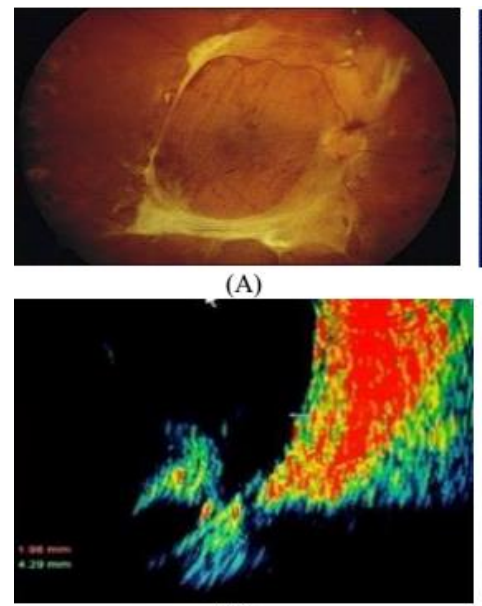

(C)

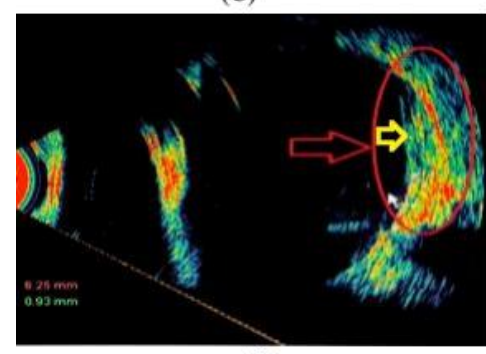

(E)
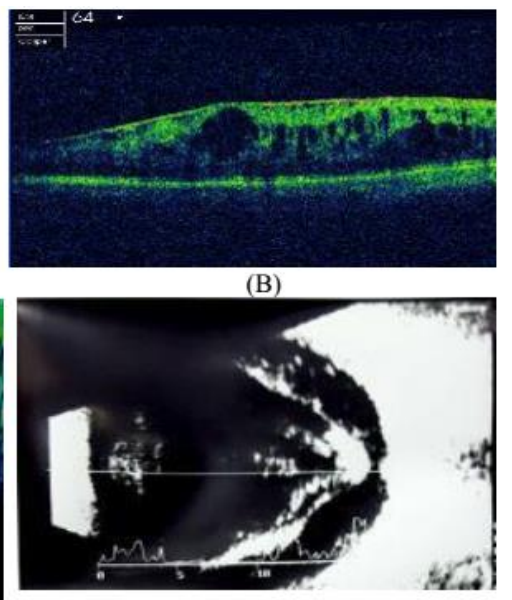

(D)

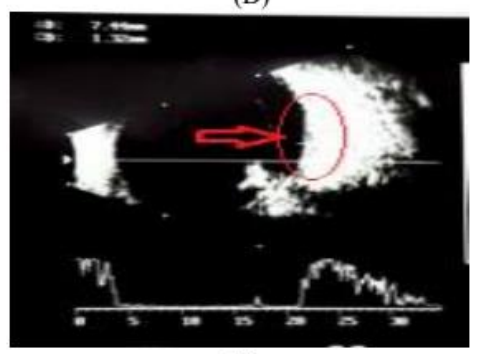

(F)

Figure 8: Rt PDR with TERM This female patient 62 years old, diabetic 21 years ago, presented by diminution of vision and by dilated funduscopy using indirect ophthalmoscopy and stereoscopic bio microscopy she had grade 1 vitreous hg, with aggressive tractional ERM. with TRD (A) fundus colored photo shows sever PDR with TERM (B) OCT shows TERM with macular bucker and tractional RD In fig (C) with $20 \mathrm{MHz}$ U $\backslash S$ we can see just the site of VMT with minimal viewing of vitreous Hg nor ERM, however on fig (D) by conventional UIS we could see that clearly. On the other hand in fig (E) we can detect the extensions of the ERMs and the site of tractional macular detachment \{arrow head\}, which couldn't differentiated well by conventional one in fig $(\mathbf{F})$. 
In group (III) of patients with (Optic nerve head disorders) we found that both of $20 \mathrm{MHz}$ US and 12.5 MHz US could easily detect the optic disc pathology with accepted quality, There was statistically total insignificant increase of pathology detection and qualification of Optic nerve head disorders 9 times by clinical + OCT-FFA more than by $20 \mathrm{MHz}$ US $(\mathrm{OR}=9 ; \mathrm{P}>0.05)$.

Table 3: Comparing pathology qualification of optic nerve disorders by each method of investigation using odds ratio and Forest plot in the study group ( $\mathrm{n}=9$ cases).

\begin{tabular}{|l|c|c|c|c|c|}
\hline \multirow{2}{*}{$\begin{array}{c}\text { Investigation } \\
\text { (Vitreo macular disorders) }\end{array}$} & \multicolumn{2}{|c|}{ Pathology qualification } & \multirow{2}{*}{ Odds ratio (OR) } & \multirow{2}{*}{ 95\% CI } & \multirow{2}{*}{ P.value } \\
\cline { 2 - 4 } & Yes & No & & & \\
\hline OCT-FFA & $9 / 9$ & $0 / 9$ & 361 & 6.4 to 20145 & $\mathbf{0 . 0 0 4 1}$ \\
\hline $20 \mathrm{MHz}$ US & $7 / 9$ & $2 / 9$ & 12.250 & 1.32 to 113.06 & $\mathbf{0 . 0 2 7 1}$ \\
\hline $12.5 \mathrm{MHz}$ US & $7 / 9$ & $2 / 9$ & 12.250 & 1.32 to 113.06 & $\mathbf{0 . 0 2 7 1}$ \\
\hline Total (fixed effects) & $23 / 27$ & $4 / 27$ & 21.790 & 5.62 to 84.488 & $<\mathbf{0 . 0 0 1}$ \\
\hline Total (random effects) & $23 / 27$ & $4 / 27$ & 20.516 & 3.99 to 105.25 & $<\mathbf{0 . 0 0 1}$ \\
\hline
\end{tabular}

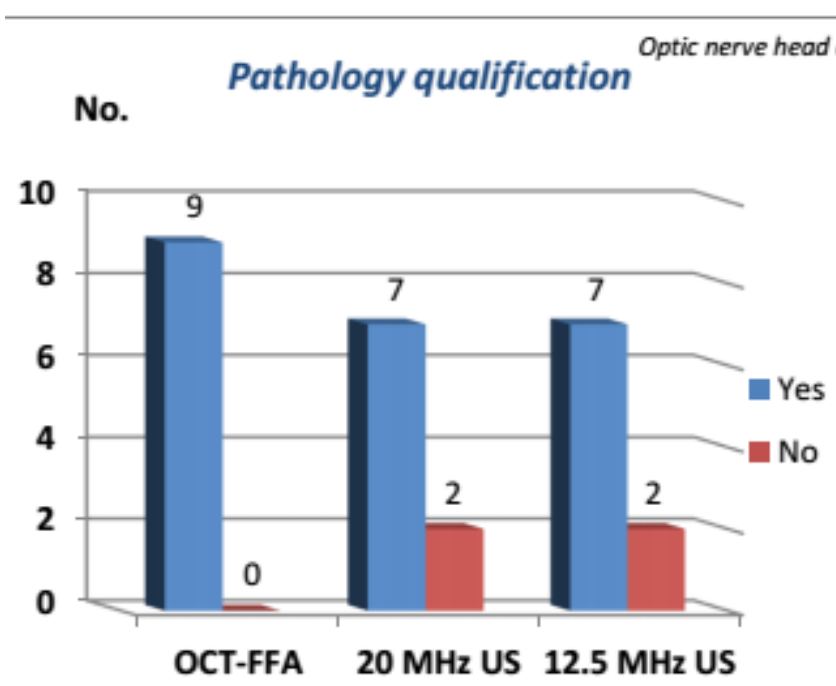

Fig. (9). Column chart comparing pathology qualification of optic nerve disorders by each method of investigation.

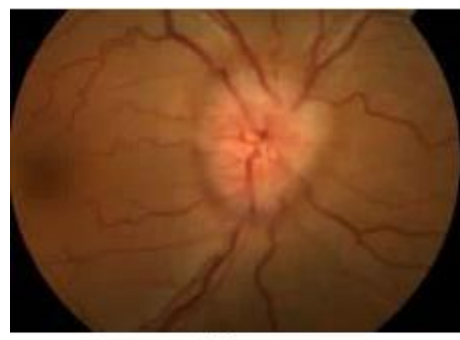

(A)

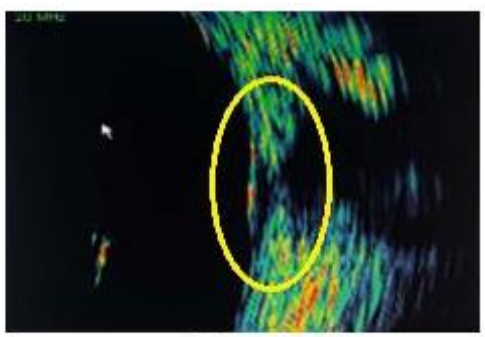

(B)

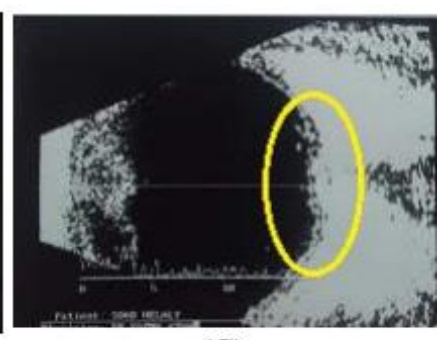

(C)

Fig. (10) Female Pt $45 y$ years with pseudo tumor cerebri\& bilateral disc edema (papilledema) (A) fundus picture of both eyes shows hyperemic swollen disc with ill-defined margins and obliterated cup with dilated tortious BVs.(B), (C) are high resolution $20 \mathrm{MHz}$ and conventional $12.5 \mathrm{MHz}$ UIS respectively, they could easily side by side detects the elevated swollen disc, with multiple small hypo-echoic fluid filled spaces resembling disc edema.

In group (IV) of patients with (Choroidal disorders) we found that:

According to pathology detection each type US was useful in pathology detection and there was no statistically difference between each of them. However, about the pathology qualification the clinical diagnosis augmented by OCT and FFA was more valuable than both types of US but statistically There was statistically total insignificant increase of pathology qualification of Choroidal disorders 9 times by OCT- 
FFA more than by $20 \mathrm{MHz}$ US (OR=9; P > 0.05). There was also there was statistically total insignificant increase of pathology qualification of Choroidal disorders 1.828 times by $20 \mathrm{MHz}$ US more than by $12.5 \mathrm{MHz}$ $\mathrm{US}(\mathrm{OR}=1.828 ; \mathrm{P}>0.05)$.

Table 4: Comparing pathology qualification of choroidal disorders by each method of investigation using odds ratio and Forest plot in the study group ( $\mathrm{n}=9$ cases).

\begin{tabular}{|l|c|c|c|c|c|}
\hline \multirow{2}{*}{$\begin{array}{c}\text { Investigation } \\
\text { (Choroidal disorders) }\end{array}$} & \multicolumn{2}{|c|}{ Pathology qualification } & \multirow{2}{*}{ Odds ratio (OR) } & \multirow{2}{*}{ 95\% CI } & \multirow{2}{*}{ P.value } \\
\cline { 2 - 3 } & Yes & No & & & \\
\hline OCT-FFA & $8 / 8$ & $0 / 8$ & 289 & 5.1 to 16318.8 & $\mathbf{0 . 0 0 5 9}$ \\
\hline 20 MHz US & $4 / 8$ & $4 / 8$ & 1 & 0.141 to 7.099 & 1 \\
\hline $12.5 \mathrm{MHz}$ US & $3 / 8$ & $5 / 8$ & 0.360 & 0.047 to 2.725 & 0.3226 \\
\hline Total (fixed effects) & $15 / 24$ & $9 / 24$ & 2.164 & 0.798 to 5.872 & $\mathbf{0 . 1 2 9}$ \\
\hline Total (random effects) & $15 / 24$ & $9 / 24$ & 2.865 & 0.12 to 63.596 & 0.506 \\
\hline
\end{tabular}

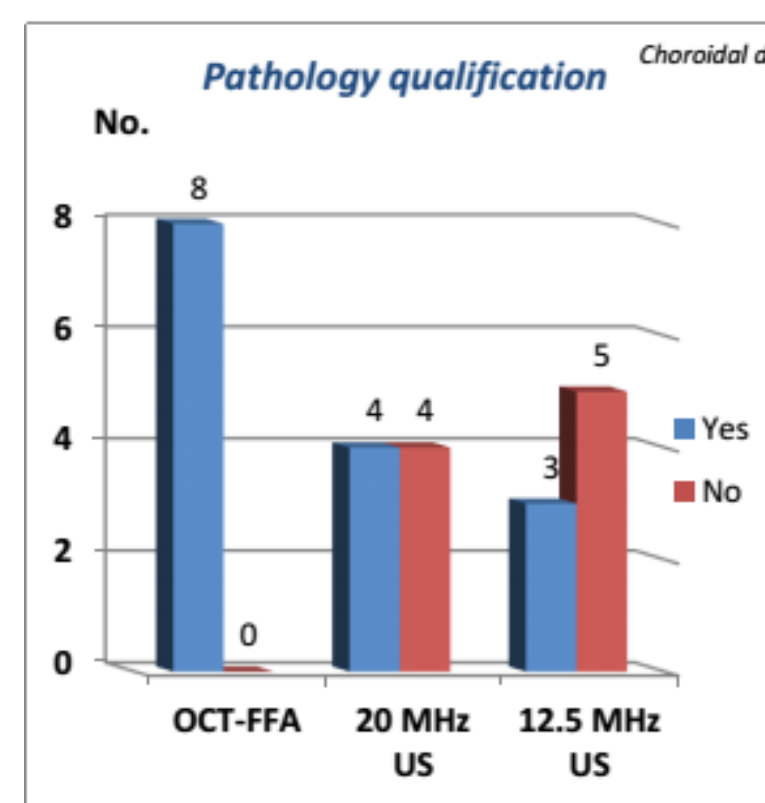

Fig. (11). Column chart comparing pathology qualification of choroidal disorders by each method of investigation

\section{DISCUSSION}

In the presenting study, we assessed the accuracy and reliability of the $20 \mathrm{MHz}$ US in the evaluation of different posterior segment pathologies, side by side with conventional 12.5 MHz US, by the help of clear media in our patients we keep the clinical diagnosis augmented by the OCT and FFA Is the gold standard. We classified the patients according to their pathology into 4 main groups.

In the 1st group of the study (retinal and vascular disorders) regarding quality of the pathology detection our results agrees with Coleman and Ronald (2). who proved that for clinical evaluation of retinal pathologies such as

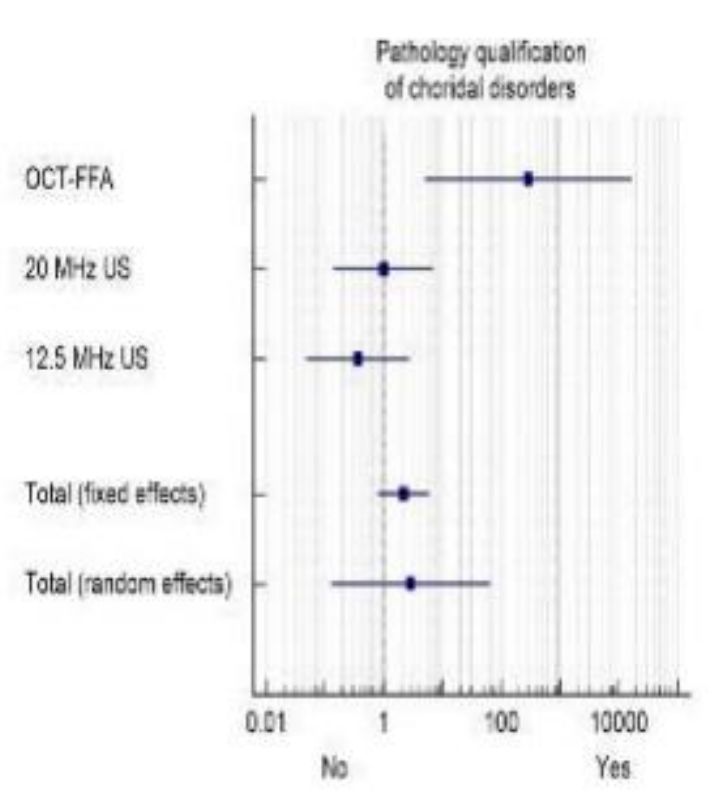

Fig. (12). Forest plot chart comparing pathology qualification of choroidal disorders by each method of investigation

breaks and macular holes, OCT provides better resolution and sensitivity than US. However, 20$\mathrm{MHz}$ US offers advantages in case of opaque media or peripheral location of the pathology makes OCT impracticable Regarding retinal pathology quantification There was statistically total insignificant increase of pathology quantification of Retinal and vascular disorders 5 times by OCT-FFA more than by $20 \mathrm{MHz}$ US $(\mathrm{OR}=5$, P.> 0.05). This agrees with what was reported by Singh et al.(2015) they proved that the difference between high resolution (immersion technique) was less than $10 \%$ in measuring retina and choroidal thickness (RCT) from that measured by EDI-OCT and thus could be considered as a reliable tool for these measurements when EDIOCT is impracticable. 
In the 2nd group of the study (Vitreomacular interface disorders) our results agrees with what's reported by hewick et al. (4)who reported that $20 \mathrm{MHz}$ probe has a better resolution and can be useful in detection of details of the posterior segment and in the orbital scanning. The $10 \mathrm{MHz}$ probe has better sensitivity and could be used to evaluate low intensity structures, such as those in the vitreous gel that cannot be easily detectable by a higher frequency probe.

In the 3rd group of the study (Optic nerve head disorders) we found that both of $20 \mathrm{MHz}$ US and $12.5 \mathrm{MHz}$ US could easily detect the optic disc pathology with accepted quality, There was statistically total insignificant increase of pathology detection and qualification of Optic nerve head disorders 9 times by clinical + OCT-FFA more than by $20 \mathrm{MHz}$ US (OR=9; P > 0.05).

In the 4th group of the study (Choroidal disorders) According to pathology detection each type US was useful in pathology detection and there was no statistically difference between each of them. However, about the pathology qualification the clinical diagnosis augmented by OCT and FFA was more valuable than both types of US but statistically There was statistically total insignificant increase of pathology qualification of Choroidal disorders 9 times by OCT-FFA more than by $20 \mathrm{MHz}$ US (OR=9; P > 0.05). There was also There was statistically total insignificant increase of pathology qualification of Choroidal disorders 1.828 times by $20 \mathrm{MHz}$ US more than by $12.5 \mathrm{MHz}$ US (OR=1.828; $\mathrm{P}>0.05)$.

Unfortunately, no more studies discussed the use of Immersion high resolution $20 \mathrm{MHz}$ US in any other pathologies or disorders, and so many pathologies in our study we were the firist to discuss it. Nevertheless, we could not completely cover the whole of posterior segment disorder and we were unlucky to find any case of choroidal melanoma or tumors throughout the duration of our study, but we hope that we put a step forward for other researches in this topic in the future.

\section{CONCLUSION}

We have two main characters for well diagnosis by any US tool, resolution and sensitivity, and neither the $20 \mathrm{MHz}$ nor $12.5 \mathrm{MHz}$ could combine both of them. Therefore, we recommended to combine the examination by of both of them as they are complementary to each other, the $10 \mathrm{MHz}$ probe for initial screening of the globe and for evaluation of vitreoretinal disorders, in particular the detection of posterior vitreous detachment, vitreous hemorrhage, and subtle vitreous change such as inflammatory cells, etc. The $20 \mathrm{MHz}$ probe is best utilised to better identify ocular wall and its lesions including optic nerve head, retinal and choroidal tissues in a high resolution. So, we recommend to use them in all of posterior segment lesions with opaque media, in which OCT \& FFA are of no role, as they could change the treatment plan or change the surgical decision.

\section{CONFLICTS OF INTEREST}

There are no conflicts of interest.

\section{REFERENCES}

1. Singh AD and Hayden BC (2012): Ophthalmic ultrasonography. Elsevier, pp: 1-2.

2. Coleman J and Ronald H (2004): HighResolution Ultrasonic Imaging of the Posterior Segment. The American Academy of Ophthalmology, 111:1344 1351.

3. Coleman J, Silverman R, Chabi A et al. (2006): High-resolution Ultrasonic Imaging of the Posterior Segment, Ophthalmology, 111(7):1344-57.

4. Hewick SA, Fairhead AC, Culy JC et al. (2004): A comparison of $10 \mathrm{MHz}$ and 20 $\mathrm{MHz}$ ultrasound probes in imaging the eye and orbit. Br J Ophthalmol., 88:551 555. 